See discussions, stats, and author profiles for this publication at: https://www.researchgate.net/publication/280719264

\title{
Precise classification of coastal benthic habitats using high resolution Worldview-2 imagery
}

Conference Paper · July 2015

DOI: 10.1109/IGARSS.2015.7326269

CITATIONS

3

4 authors, including:

J. Marcello

Universidad de Las Palmas de Gran Canaria

76 PUBLICATIONS 694 CITATIONS

SEE PROFILE

Javier Martin Abasolo

3. Universidad de Las Palmas de Gran Canaria

27 PUBliCATIONS 202 CITATIONS

SEE PROFILE

Some of the authors of this publication are also working on these related projects:

Project ARTEMISAT View project

Project H2020 Impressive View project
READS

232

Francisco Eugenio

Universidad de Las Palmas de Gran Canaria

69 PUBLICATIONS 601 CITATIONS

SEE PROFILE 


\title{
PRECISE CLASSIFICATION OF COASTAL BENTHIC HABITATS USING HIGH RESOLUTION WORLDVIEW-2 IMAGERY
}

\author{
J. Marcello ${ }^{1}$, F. Eugenio ${ }^{1}$, F. Marques $^{2}$, J. Martín $^{1}$ \\ ${ }^{1}$ Instituto de Oceanografía y Cambio Global (IOCAG). Universidad de Las Palmas de Gran Canaria. \\ Spain.E-mails: javier.marcello@ulpgc.es, francisco.eugenio@ulpgc.es, \\ javier.martin.abasolo@gmail.com \\ ${ }^{2}$ Signal Theory and Communications Department. Universitat Politecnica de Catalunya \\ BarcelonaTECH. \\ Spain. E-mail: ferran.marques@upc.edu
}

\begin{abstract}
The analysis of the seafloor in shallow waters using remote sensing imagery at very high spatial resolution is a very challenging topic due to the minimum signal level received; the presence of noisy contributions from the atmosphere, solar reflection, foam, turbidity and water column; and the limited spectral information available for the classification at such depths that impedes, for example, the extraction of vegetation indices. In this complex scenario we have developed a mapping methodology that involves the precise application of pre-processing techniques and the use of efficient classification algorithms. In particular, after a detailed assessment, support vector machines achieved the best performance using the appropriate kernel and parameters. Two natural areas located at the Canary Islands (Spain) have been selected for their benthic habitats richness and specially for their preservation of highly protected seagrass regions.
\end{abstract}

Index Terms-Worldview-2, benthic classification, SVM, seagrass.

\section{INTRODUCTION}

Benthic habitat maps provide useful information for the management of coastal ecosystems and are used in various research and monitoring activities. Common techniques to map benthic habitats have been field sampling and aerial photography. However, they require time, are labor intensive, expensive and limited over remote areas. For all these, satellite imagery is becoming important for coastal and marine environments monitoring. Nowadays, very high resolution (VHR) data offer new opportunities [1]; however, better spatial resolution does not necessarily benefit classification performance and, in consequence, the development of robust classification techniques has been an intense research area in the former years.
The mapping of benthic habitats is a very challenging problem as a consequence of the minimum level of the signal received by the sensor in comparison with other noisy contributions present in the images, of various contributing factors (atmosphere, solar reflection, foam, turbidity, water column, etc.), and of the limited spectral information available for the classification because, depending of the depth to analyze, only bands around the blue and green can be useful. In this complex context, we have developed a methodology for the robust classification of shallow environments using VHR imagery. In particular, Worldview-2 (WV-2) imagery has been selected due to its excellent spatial resolution and the availability of 8 bands.

\section{MAPPING METHODOLOGY}

\subsection{Data}

Coastal waters around Corralejo (Fuerteventura Island) and Maspalomas (Gran Canaria Island) areas belong to the UNESCO's World Heritage natural protected areas of the Canary Islands (Spain). To perform the study, WorldView-2 ortho-ready multispectral images of Corralejo and Maspalomas were used. Figure 1 shows the geographic location of such places and the WV-2 true color image used in this work.

Ground truth data to train and validate the classification algorithms was obtained by reference maps provided mainly by the Canarian Government [2, 3]. An in-situ field campaign was also conducted at Maspalomas to collect seafloor information and to measure water quality parameters.

\subsection{Pre-processing for benthic mapping}

A critical step for benthic classification is the pre-processing of Worldview-2 images. It involves radiometric conversion, atmospheric correction, sunglint removal, and correction for the water column. 

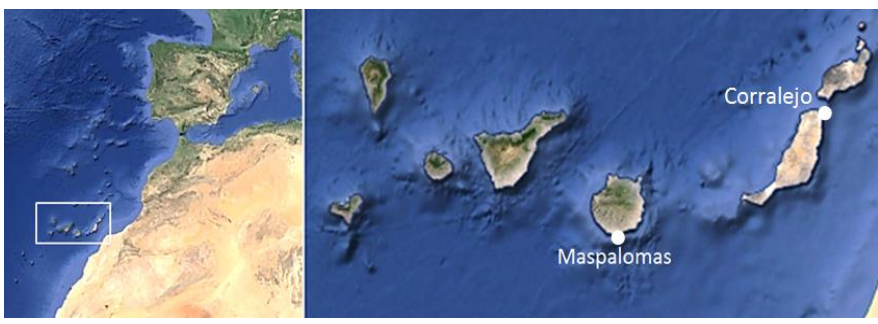

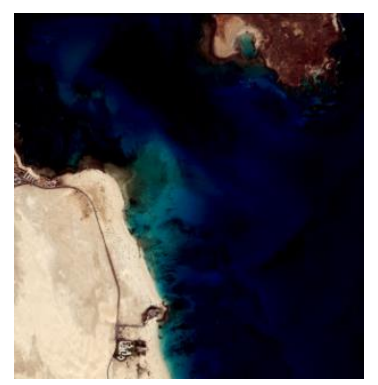

(b) (a)

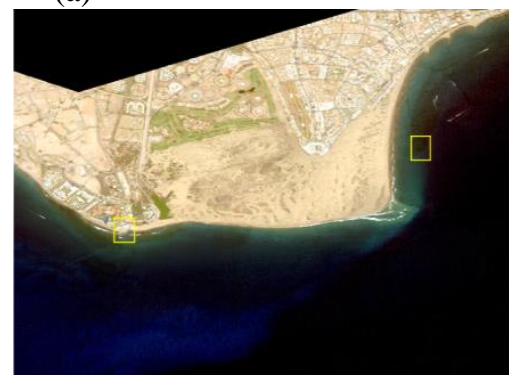

(c)
Figure 1. Areas of study: (a) geographic location, (b) Worldview-2 color composite of Corralejo (October 28, 2010) and (b) Worldview-2 color composite of Maspalomas (August 11, 2013).

After a detailed assessment, comparing the retrieved reflectance with ground-based measurements for a variety of targets, we have applied the $6 \mathrm{~S}$ atmospheric model with the appropriate input parameters [4]. In addition, sunglint adds a noisy radiation component to the signal registered by the sensor, typically much higher than the water leaving signal in all spectral bands, saturating pixel values. In our work we have applied an improved algorithm for sunglint removal based on combined physical and image processing techniques [4].

Afterward, water column correction is necessary to take into account the exponential attenuation of the reflectance due to absorption and scattering. The degree of attenuation varies with the wavelength and the spectral signature of a benthic habitat changes with increasing depth. To reduce these problems, we have applied the methodology included in [5].

Finally, pan-sharpening techniques [6] were assessed to increase the spatial resolution with minimum spectral distortion with the goal to improve the classification accuracy. Unfortunately, results were very similar, mainly due to the fact that the PAN band can only provide useful information at very shallow waters. Figure 2 includes examples of the Gram-Schmidt pansharpening for specific sea and land areas (yellow squares in Figure 1c). A clear spatial improvement is appreciated in land but it is not as clear in the delimitation of benthic habitats.

\subsection{Classification of benthic habitats}

After the pre-processing steps, images can be more reliably classified. Many methods have been developed to classify digital images using the spectral, spatial or temporal properties of the objects present in the images. However, the seabed classification in very shallow non-tropical waters remains still as a challenging topic.

In our analysis we have assessed the following classifiers [7]: Mahalanobis Distance (MH), Maximum Likelihood (ML), Spectral Angle Mapper (SAM) and Support Vector Machines (SVM) using the appropriate kernel type and parameters.

In particular, support vector machines (SVM) [8] have proven to be very effective in solving complex remote sensing classification problems, mainly due to the fact that this technique does not require an estimation of the statistical distribution of classes and to its ability to handle limited amount or quality of training samples. This last property is very useful in seafloor mapping as acquiring ground truth data is generally an expensive process.

In addition to the spectral signatures provided by the sensor, we also tested the inclusion of context information to increase the performance of the classification. Thus, we considered adding, to the multispectral information, textural data (variance and entropy from the grey-level co-ocurrence matrix) but such spatial information was not finally included as an improvement was not achieved.
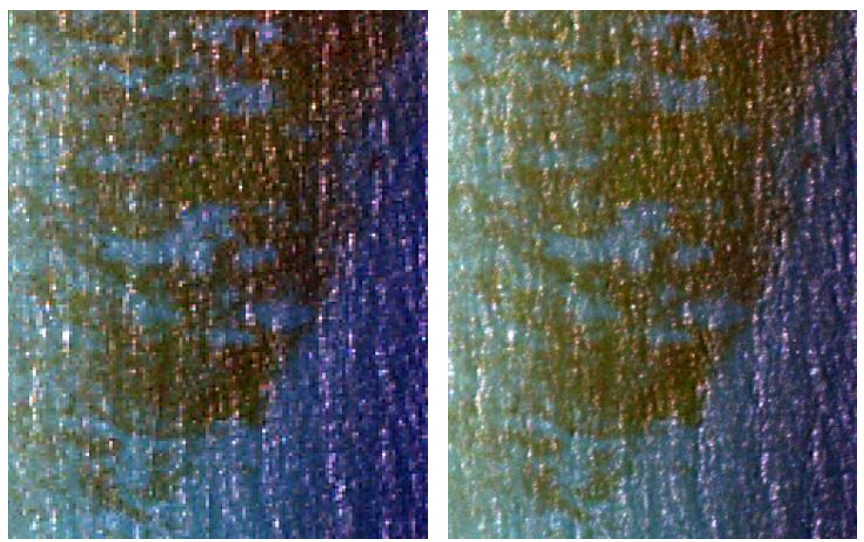

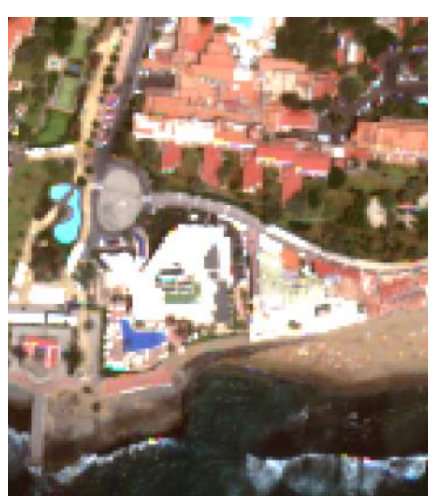

(a)

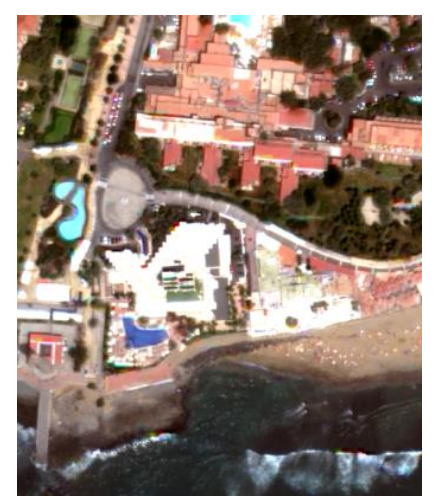

(b)
Figure 2. Worldview-2 color composites: (a) Multispectral bands and (b) Gram-Schmidt pansharpened bands. 


\section{RESULTS}

\subsection{Data}

After performing the pre-processing steps described in Section 2.2, we have analyzed the various classifiers mentioned in Section 2.3 using the same training regions. First, we have examined the spectral separability of the classes by means of the Jeffries-Matusita distance. Next, we applied the four supervised methods previously indicated. Finally, in order to assess the classification accuracy, apart from visual evaluation, we have computed the confusion matrix and the kappa coefficient using a new set of test regions.

After the assessment of both natural protected areas, SVM provided the highest accuracy followed by ML, MH and SAM algorithms (kappa values for Corralejo/Maspalomas are 0.68/0.74 for ML and 0.71/0.79 for SVM). Figures 3 and 4 provide a visual comparison of the maps generated by the different classifiers for Corralejo and Maspalomas, respectively. Class colors have been selected as follows: vegetation in green, sandy bottoms in yellow and rocks in brown (Corralejo) or purple (in Maspalomas to match the color contained in the reference map). Satellite classification is only possible for shallow waters. Thus, only thematic information for water depths below $25 \mathrm{~m}$ has been estimated and a blue mask has been applied for the remaining waters.

It is important to emphasize that SVM parameters were thoroughly adjusted as they affect the classification performance. In particular, the kernel type, error penalty, and gamma terms were the parameters analyzed [9, 10]. After the evaluation, the gaussian radial basis kernel was selected as it provided improved results. Then, the gridsearch methodology was used to choose the appropriate error penalty and Gamma values. The points were chosen on a logarithmic scale, initially using a coarse grid and next conducting a fine search around the maximum accuracy values.

\section{CONCLUSIONS}

Given the importance of the coastal ecosystems for life quality and the global climate, systematic and efficient information is important for the monitoring of such protected areas.

Satellite-based imaging systems with bands within the visible and near infrared spectrum can provide information at spatial scales needed to implement conservation actions for singular coastal locations as those included in our work (Corralejo and Maspalomas marine protected areas). In this context, Worldview-2 data were selected as it provides various high-resolution spectral bands at short wavelength that can reach the seabed.

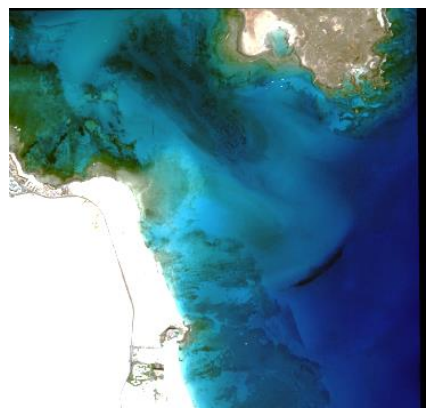

(a)

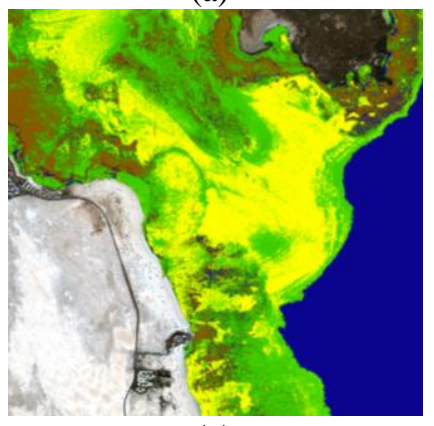

(c)

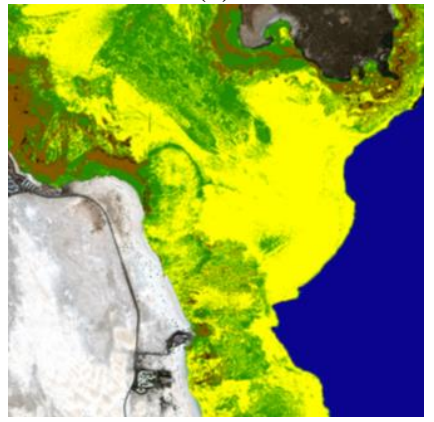

(e)

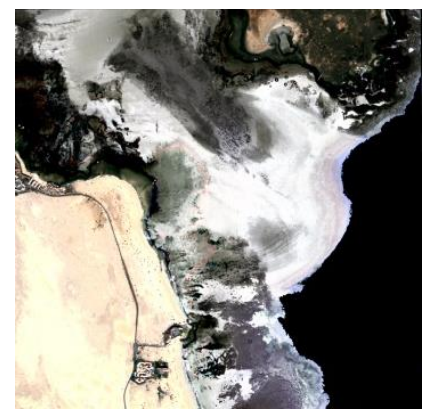

(b)

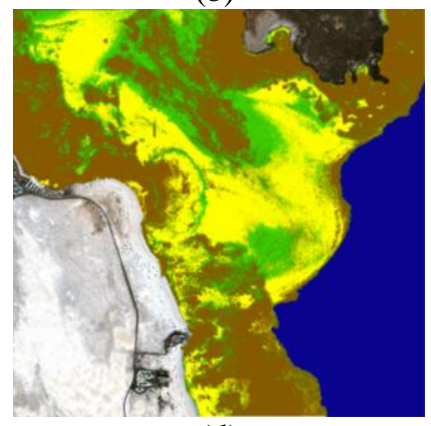

(d)

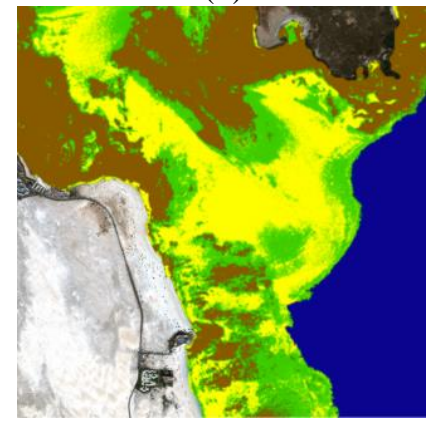

(f)
Figure 3. Classification results for Corralejo: (a) WV-2 image with histogram enhancement for a better seafloor visualization, (b) reference map, (c) Mahalanobis Distance, (d) Maximum Likelihood, (e) Spectral Angle Mapper and (f) Support Vector Machines.

The complex pre-processing of the very high resolution Worldview-2 images has been implemented and applied involving the radiometric, atmospheric, sunglint and water column corrections. Then, we have assessed the performance of different supervised classification algorithms when applied to diverse combinations of spectral and spatial information with the aim to obtain the best methodology to generate reliable benthic maps.

We have generated the corresponding training and test regions using data from official maps, transects and in-situ observations. After applying reliable classification algorithms (Mahalanobis Distance, Maximum Likelihood, Spectral Angle Mapper and Support Vector Machines), we can conclude that SVM provides the best performance with acceptable accuracies taking into account that mapping of benthic habitats is very challenging due to the noisy 
contributions (atmosphere, sunglint and water column), the low signal received and the limited spectral information available for the classification.

The SVM kernel type, error penalty, and gamma parameters were tuned accordingly to produce the best overall accuracy.

We also evaluated the improvement attained applying pansharpening algorithms or incorporating additional textural bands to the original spectral information; however results were not fully satisfactory and, thus, the application of the pre-processing and mapping methodology to the original bands was selected.

We are currently working on advanced object-based classification strategies to improve the final thematic maps obtained.

\section{ACKNOWLEDGEMENTS}

This work has been supported by the ARTEMISAT (CGL2013-46674-R) project, funded by the Spanish Ministerio de Economía y Competitividad.

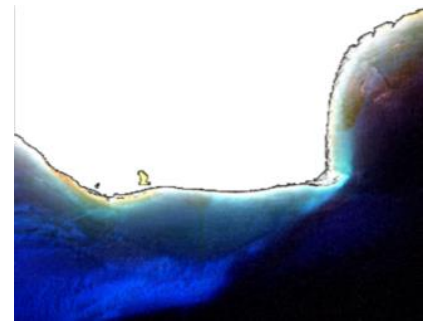

(a)

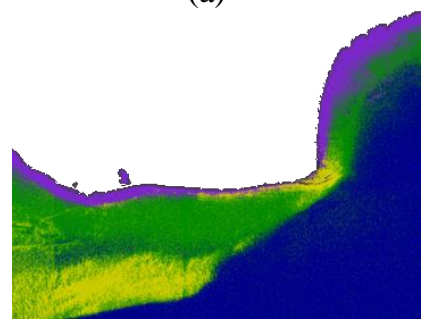

(c)

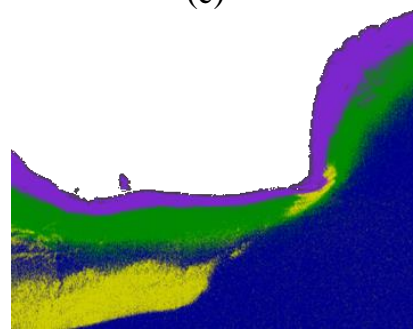

(e)

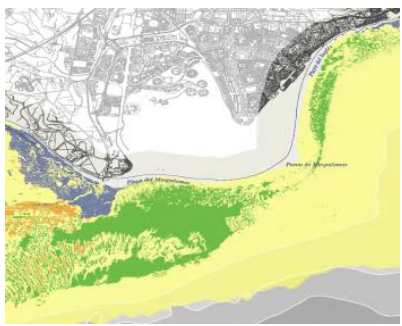

(b)

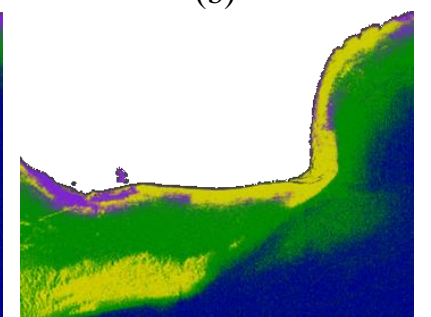

(d)

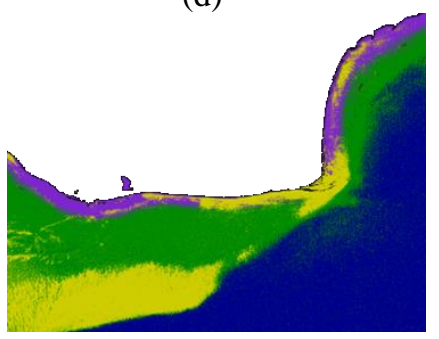

(f)
Figure 4. Classification results for Maspalomas: (a) masked WV-2 image with histogram enhancement for a better seafloor visualization, (b) reference map, (c) Mahalanobis Distance, (d) Maximum Likelihood, (e) Spectral Angle Mapper and (f) Support Vector Machines.

\section{REFERENCES}

[1] M. Lyons, S. Phinn and C. Roelfsema, "Integrating Quickbird multi-spectral satellite and field data: Mapping bathymetry, seagrass cover, seagrass species and change in Moreton bay, Australia in 2004 and 2007," Remote Sensing, vol. 3, pp. 42-64, 2011.

[2] Gobierno de Canarias. Plan Regional de Ordenación de la Acuicultura de Canarias. Tomo I: Memoria de Información del Medio Natural Terrestre y Marino, December 2013.

[3] Martinez, J., Análisis espacio-temporal de las praderas de Cymodocea nodosa (Ucria) Ascherson en la isla de Gran Canaria, Master Thesis, Universidad de Las Palmas de Gran Canarias, Dec. 2011.

[4] F. Eugenio, J. Martin, J. Marcello, and E. Fraile-Nuez, "Environmental monitoring of El Hierro Island submarine volcano, by combining low and high resolution satellite imagery," International Journal of Applied Earth Observation and Geoinformation, vol. 29, pp. 53-66, 2014.

[5] Martin, J., F. Eugenio, and J. Marcello Estudio, Implementación y validación de algoritmos para la cartografía de vegetación béntica (sebadales) a partir de las imágenes corregidas del satélite Worldview 2, Informe Final de la Fase 5 del convenio de colaboración Observatorio Ambiental de Granadilla y Universidad de Las Palmas de Gran Canaria, Febrero 2013.

[6] G. Vivone, L. Alparone, J. Chanussot, M. Dalla Mura, A. Garzelli, G. Licciardi, Restaino and L. Wald, "A Critical Comparison Among Pansharpening Algorithms," IEEE Transactions on Geosciences and Remote Sensing, v. 53, n.5, 2015.

[7] Tso, B. and P.M. Mather, Classification Methods for Remotely Sensed Data, Taylor and Francis Inc., New York, 2009.

[8] G. Mountrakis, J. Im and C. Ogole, "Support vector machines in remote sensing: A review," ISPRS Journal of Photogrammetry and Remote Sensing, vol. 66, pp. 247-259, 2011.

[9] T. Kavzoglu and I. Colkesen, "A Kernel Functions Analysis for Support Vector Machines for Land Cover Classification," International Journal of Applied Earth Observation and Geoinformation, vol. 11-5, pp. 352-359, 2009.

[10] X. Yang, "Parameterizing Support Vector Machines for Land Cover Classification," Photogrammetric Engineering and Remote Sensing, vol. 77- 1, pp. 27-37, 2011. 